\title{
Improving the Estimation of Meal-Time Insulin Dose Based On the Glycaemic Load of a Meal in Children with Type 1 Diabetes on Insulin Pump Therapy: A Randomized Study
}

\author{
Lidia Groele ${ }^{1}$, Dominik Golicki ${ }^{2}$, Marlena Błazik ${ }^{3}$ and Ewa Pańkowska ${ }^{4}$ \\ ${ }^{1}$ Department of Paediatrics, Medical University of Warsaw, Warsaw, Poland \\ ${ }^{2}$ Department of Experimental and Clinical Pharmacology, Medical University of Warsaw, Warsaw, Poland \\ ${ }^{3}$ The Mother and Child Institute, Warsaw, Poland \\ ${ }^{4}$ Diabetology Clinic, The Mother and Child Institute, Warsaw, Poland
}

\begin{abstract}
Background: The aim of the study was to assess whether an Insulin-Carbohydrate Ratio (ICR) increased by $30 \%$ per meal with a high glycaemic load allows postprandial glucose excursion to be maintained in control in children with type 1 diabetes.

Methods: A total of 70 children on insulin pump therapy participated in the study. They were stratified into age groups: 4-7 years of age, 8-12 years of age and above 12 years of age. The experimental Group (A) received an insulin dose based on individualised ICR increased by $30 \%$ for breakfast containing cornflakes with milk. The control group (B) received an insulin dose based on standard individualised ICR. The glycaemic load of the meal was adjusted to the age group $(24,3 ; 32,4 ; 40,5)$. We assessed metabolic effects such as the area under the glucose curve (AUC), glucose increment, risk of hyperglycaemia and hypoglycaemia using two methods: blood glucose concentration (at $0,15,30,45,60,90$ and 120 minutes) and a continuous subcutaneous glucose measurement system (for 3 hours postprandially).
\end{abstract}

Results: A significant glucose rise was noted in the control group B $(p=0.03)$. Over the postprandial 180 minutes, AUC was significantly lower $(p=0.02)$ in the experimental group but only in children aged $8-12$. The frequency of hypoglycaemic events was not statistically significant during postprandial observation $(p=0.75)$.

Conclusion: Increasing the ICR by $30 \%$ for a meal with high GL can reduce the glucose excursion without increasing the risk of hypoglycaemia in children with type 1 diabetes treated with insulin pump therapy.

Keywords: Diabetes type 1; Glycaemic load; Postprandial glycaemia; Prandial insulin dose; Insulin pump

\section{Background}

Functional Insulin Therapy (FIT) reduces the risk of diabetic late complications, improves the quality of treatment as well the quality of life, and is a recommended method for treating Type 1 Diabetes (T1D) patients $[1,2]$. In terms of postprandial glucose control, meal insulin dosage based on carbohydrate counting, the 'golden standard' in FIT, is unsatisfactory probably due to many factors influencing postprandial glucose excursion. Food counting and the exchange system in which grams of carbohydrates are expressed as a unit of bread were introduced several years ago [3]. This system is not always sufficiently effective. Therefore, factors other than only carbohydrate grams are considered in meal insulin adjustment. According to the American Diabetes Association (ADA) both Glycaemic Load (GL) and Glycaemic Index (GI) should be considered when choosing carbohydrate products [1]. Glycaemic Load (GL), apart from the glycaemic index, takes into account carbohydrate content in the meal. Although products with high glycaemic load are not recommended for children, due to their physiological development, children need to consume carbohydrate meals which can be easily and quickly absorbed. Unfortunately, the availability of fast food makes it easy for children to consume this type of meal far too often. It is also difficult to limit the consumption of such products among children with type 1 diabetes, especially among small children who have a tendency to consume easily absorbed carbohydrate products $[4,5]$.

In the light of current knowledge, meals with a high GL cause postprandial hyperglycaemia in people with type 1 diabetes [6].
However, there is no data on its influence on insulin requirement. The dose of insulin is calculated on the basis of the amount of the carbohydrate and insulin-carbohydrate ratio [2]. There is no research on the safety and efficacy of insulin administration depending on the GL of a meal among patients with T1D. Studies exist which try to establish the optimal insulin adjustment for a meal in FIT among patients with T1D $[7,8]$.

In the study, we assumed that for products with high GL, the ICR should be increased by $30 \%$ to obtain postprandial normoglycaemia. In formulating our hypothesis, we took into account the results of a study on the correlation between the GL of a meal and postprandial glycaemia in healthy subjects which show about a 30\% higher plasma glucose response to the consumption of breakfast cereal with low and high glycaemic indexes [9]. Moreover, a higher serum glucose and insulin AUC over 2 and 5 hours for the high- versus low-glycaemic

*Corresponding author: Lidia Groele, Department of Paediatrics, Medical University of Warsaw 01-184 Warsaw, Działdowska 1 str., Poland, Tel: 00482245 23 284; Fax: 0048224523 309; E-mail: Igroele@wp.pl

Received June 28, 2014; Accepted September 16, 2014; Published September 24, 2014

Citation: Groele L, Golicki D, Błazik M, Pańkowska E (2014) Improving the Estimation of Meal-Time Insulin Dose Based On the Glycaemic Load of a Meal in Children with Type 1 Diabetes on Insulin Pump Therapy: A Randomized Study. J Diabetes Metab 5: 435 doi:10.4172/2155-6156.1000435

Copyright: $\odot 2014$ Groele L, et al. This is an open-access article distributed under the terms of the Creative Commons Attribution License, which permits unrestricted use, distribution, and reproduction in any medium, provided the original author and source are credited. 
Citation: Groele L, Golicki D, Błazik M, Pańkowska E (2014) Improving the Estimation of Meal-Time Insulin Dose Based On the Glycaemic Load of a Meal in Children with Type 1 Diabetes on Insulin Pump Therapy: A Randomized Study. J Diabetes Metab 5: 435 doi:10.4172/21556156.1000435

Page 2 of 5

index in obese women was also observed (above $30 \%$ up to almost twofold).

\section{Method}

This randomised, open-label study, approved by the Ethics Committee of the Medical University of Warsaw, was conducted in the Department of Paediatrics, the Medical University of Warsaw, Poland, in accordance with Good Clinical Practice (Figure 1). Written informed consent, including guardian consent for paediatric participants, was obtained from each participant. The inclusion criteria were as follows: type 1 diabetes, $\mathrm{HbAlc}<8 \%$, out of the honeymoon phase (defined by the disease duration over one year and an insulin requirement of over $0.5 \mathrm{unit} / \mathrm{kg} /$ day), non-treated with any medications influencing carbohydrate metabolism (e.g. glucocortycosteroids), no acute or chronic digestive system diseases, body mass index below the $95^{\text {th }}$ percentile, treated with continuous subcutaneous insulin infusion (CSII), and age between 4 and 18 years. The participants were stratified into three age subgroups: group I (4-7 years), group II (8-12 years) and group III (above 12 years).

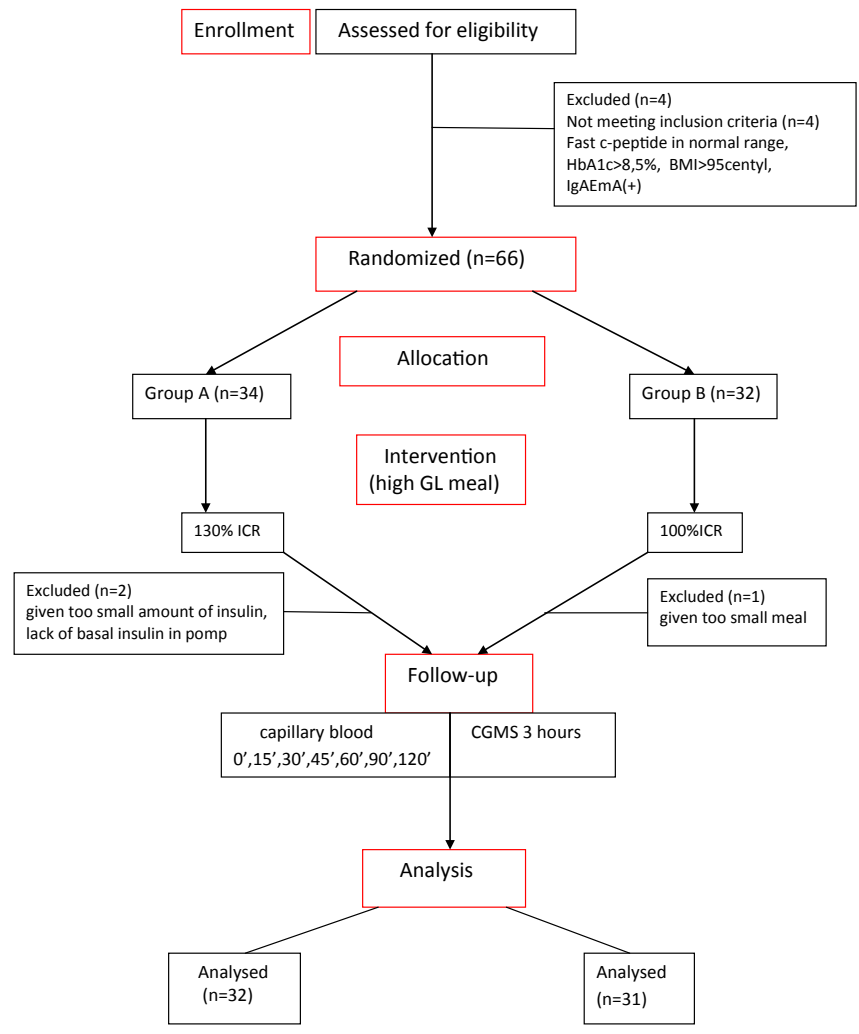

Figure 1: Flow diagram of the stages of the study.

\section{Procedure}

We measured blood glucose levels in groups $\mathrm{A}$ and $\mathrm{B}$. The samples were collected at $0,15,30,45,60,90$ and 120 minutes after the meal. Glucose levels were assessed using the hexokinase method. Additionally, postprandial glucose excursion was monitored by means of a Continuous Subcutaneous Glucose Measurement System (CGMS) for 3 hours after the meal.

\section{Meal}

The study meal served for breakfast to all participants contained Nestle cornflakes with $2 \%$ UHT milk as an extender. The size of the meal was adjusted to the age of the child (Table 1). The participants consumed the meal over 15 minutes under the supervision of a nurse when the fasting glucose level was between 70 and $140 \mathrm{mg} / \mathrm{dL}$ after an 8-hour night break.

\section{Insulin dose}

After enrolment, all participants had a nine-point glucose profile assessed in-hospital for 3 consecutive days and had their individualised breakfast ICR calculated. They were then randomised and the study was conducted. We used individualised ICR increased by $30 \%$ for cornflakes and standard individualised ICR for milk in group A. In group B, we used standard individualised ICR for both cornflakes and milk. Insulin was administered 5-10 minutes before the meal.

\section{Metabolic outcomes}

Postprandial glucose excursion was assessed using the following parameters:

- the Area Under The Curve (AUC) of postprandial glucose level over 180 minutes,

- Postprandial Glucose Excursions (PPGE), defined as the change in glucose concentration from before the meal to Peak Postprandial Glucose (PPG),

- hyperglycaemic (cut-off point $180 \mathrm{mg} / \mathrm{dL}$ ),

- hypoglycaemic episodes over time between groups,

- hypoglycaemic events,

- hypoglycaemia defined as $\mathrm{BG}<70 \mathrm{mg} / \mathrm{dL}$ (10).

\section{Insulin requirements}

Total daily prandial and basal insulin doses were downloaded from the insulin pump memory using the Solutions Pumps \& Meters Software Model MMT-7311 version 7.0 MiniMedMetronic for MiniMed 508, 712, 715, 722, and the Accu-Chek Smart V Roche Diagnostics for the Accu-Chek.

\begin{tabular}{|c|c|c|c|c|c|c|}
\hline & \multicolumn{2}{|c|}{ I group (4-7yr) } & \multicolumn{2}{|c|}{ II group (8-12yr) } & \multicolumn{2}{|c|}{ III group (>12yr) } \\
\hline & Nestle cornflakes $40 \mathrm{~g}$ & $2 \%$ UHT milk $-100 \mathrm{~mL}$ & Nestle cornflakes $53 \mathrm{~g}$ & $2 \%$ UHT milk $-150 \mathrm{~mL}$ & Nestle cornflakes $67 \mathrm{~g}$ & $2 \%$ UHT milk $-200 \mathrm{~mL}$ \\
\hline $\mathrm{CU}$ & 3 & 0.5 & 4 & 0.75 & 5 & 1 \\
\hline $\mathrm{Gl}$ & 81 & 27 & 81 & 27 & 81 & 27 \\
\hline GL & 24.3 & 1 & 32.4 & 2 & 40.5 & 3 \\
\hline Total CU & \multicolumn{2}{|c|}{3.5} & \multicolumn{2}{|c|}{4.75} & \multicolumn{2}{|c|}{5} \\
\hline
\end{tabular}

CU: Carbohydete Union; GI: Glycaemic Index; GL: Glycaemic Load 
Citation: Groele L, Golicki D, Błazik M, Pańkowska E (2014) Improving the Estimation of Meal-Time Insulin Dose Based On the Glycaemic Load of a Meal in Children with Type 1 Diabetes on Insulin Pump Therapy: A Randomized Study. J Diabetes Metab 5: 435 doi:10.4172/21556156.1000435

Page 3 of 5

\section{Statistical Analysis}

\section{Sample size calculation}

With an AUC of postprandial glucose level over 180 minutes as the primary end-point, we assumed that differences of $30 \%$ between the study groups in that parameter and SD $40 \%$ was significant for the metabolic control. Using 1:1 randomisation, 'alpha' 0.05 and power ('beta') $80 \%$, it was determined that each study group should comprise 28 patients. Considering that $20 \%$ of the patients might drop out of the study, we determined the sample size to be 70 participants.

The results were analysed with the Intention-To-Treat (ITT) population. The normality of the distribution of all parameters was assessed using the Shapiro-Wilk test. The significance of the differences between the two groups was calculated using the t-Student, MannWhitney, Smirnov-Kolmogorov tests for unrelated variability, adjusting the $\mathrm{t}$-Student or Fischer test for related variables. Additionally we counted the RR and NNT numbers needed for treatment. The level of statistical significance was set in all analyses as $p<0.05$. The results were analysed in groups divided according to Intention-To-Treat analysis (ITT) and in age groups according to the protocol (PP-per protocol analysis). The statistical analysis was performed with Microsoft Office 2007 and Stats Direct Version, 2007.

\section{Result}

A total of 70 children and adolescents with type 1 diabetes mellitus participated in the study. 63 of them completed the study. 7 patients were disqualified from the study ( 3 from group A and 4 from group B) because of improper prandial insulin doses, too small meals, lack of basal insulin in the pump during the postprandial period, c-peptides above norm range, and the presence of positive endomysial antibodies class A. There were no differences between the study groups in the baseline parameters: demographic data, metabolic control or insulin requirement (Table 2).

\begin{tabular}{|l|c|c|c|}
\hline & \multicolumn{2}{|c|}{ Mean \pm SD } & \\
\hline & Group A & Group B & \\
\hline Number of patients & 32 & 31 & NS \\
\hline Female & 18 & 15 & NS \\
\hline BMI SDS & $0.4 \pm 1.0$ & $0.3 \pm 0.6$ & NS \\
\hline Duration of diabetes (years) & $6.2 \pm 3.0$ & $5.1 \pm 3.0$ & NS \\
\hline HbA1c (\%) & $7.0 \pm 0.7$ & $6.9 \pm 1.0$ & NS \\
\hline Carbohydrate-insulin ratio for breakfast (IU/1CU) & $1.4 \pm 0.5$ & $1.3 \pm 0.4$ & NS \\
\hline TDD (IU/kg bm) & $0.8 \pm 0.2$ & $0.7 \pm 02$ & NS \\
\hline Basal/ IU/24h & $37.3 \pm 12.6$ & $39.7 \pm 12.8$ & NS \\
\hline Fasting glucose (mg/dl) & $110.5 \pm 26.7$ & $111.8 \pm 25.3$ & NS \\
\hline
\end{tabular}

SD: Standard Deviation

Table 2: Characteristic of the study group (63 patients).

\begin{tabular}{|l|c|c|c|}
\hline Hyperglycaemia & Group A & Group B & \\
\hline Total number of hyperglycaemia episodes & 139 & 249 & \\
\hline Time of all hyperglycaemia episodes(min) & 695 & 1245 & \\
\hline Maximal glucose level (mg/dL) & 271 & 400 & \\
\hline Maximal period of episode (min) & 105 & 160 & \\
\hline Number of patients with $\geq 1$ episodes & $11(39 \%)$ & $16(53 \%)$ & \\
\hline & \multicolumn{2}{|c|}{ Mean \pm SD } & p-value \\
\hline Frequency episodes /patient & $5.0 \pm 7.4$ & $8.3 \pm 9.6$ & 0.45 \\
\hline Time of hyperglycaemia/patient (min) & $24.8 \pm 37.3$ & $41.5 \pm 48$ & 0.45 \\
\hline SD: Standard Deviation & \multicolumn{3}{|l}{} \\
\hline
\end{tabular}

Table 3: Frequency of postprandial hyperglycaemia over 180 minutes estimated by CGMS.

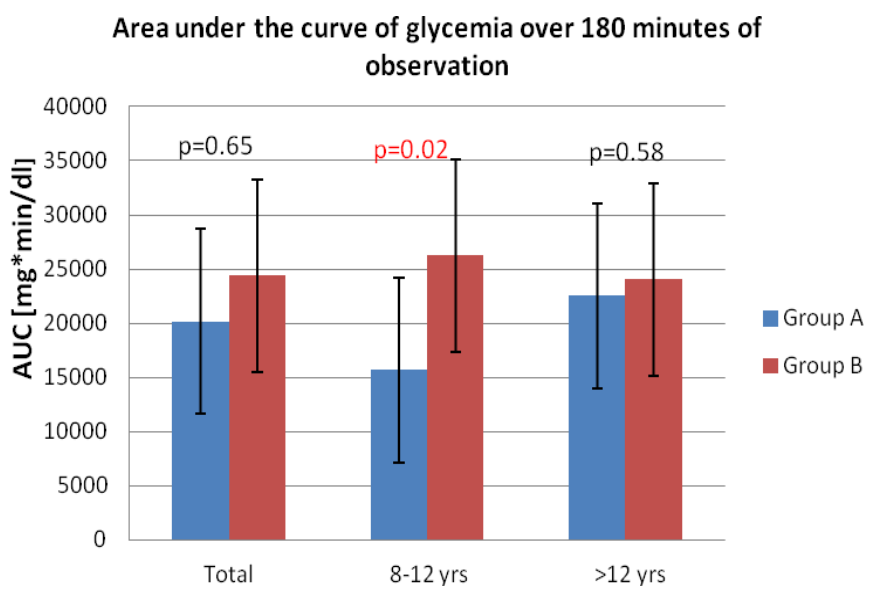

Figure 2: Area under the curve of glycaemia over 180 minutes' observation in Group A and Group B, depending on age.

\begin{tabular}{|l|c|c|c|}
\hline & \multicolumn{2}{|c|}{ Mean \pm SD } & \\
\hline & Group A & Group B & p-value \\
\hline Hypoglycaemia in capillary blood & \multicolumn{3}{|l|}{} \\
\hline Frequency episodes/patient & $0.21 \pm 0.4$ & $0.15 \pm 0.36$ & $\mathbf{0 . 4 2}$ \\
\hline Number of hypoglycaemia episodes & 7 & 5 & \\
\hline Lowest glucose level(mg/dL) & 47 & 40 & \\
\hline Hypoglycaemia in CGMS & & & \\
\hline Frequency episodes/patient & $4.5 \pm 6.3$ & $4.1 \pm 7.2$ & 0.98 \\
\hline Number of hypoglycaemia episodes & 125 & 125 & \\
\hline Lowest glucose level(mg/dL) & 40 & 40 & \\
\hline
\end{tabular}

SD: Standard Deviation

Table 4: Frequency of postprandial hypoglycaemia over 120 minutes in capillary blood and over 180 minutes in CGMS.

\section{Glucose area under the curve}

The postprandial area under the glucose curve (AUC) over 180 minutes was not statistically significant between groups $\mathrm{A}$ and $\mathrm{B}$ and the average was $20,187.3 \pm 8532.1$ vs $24,384.7 \pm 8917.1 \mathrm{mg}^{*} \mathrm{~min} /$ $\mathrm{dl}$ respectively $(\mathrm{p}=0.065)$, but it tended to produce a greater response in the whole control group. Among children aged 8-12 years, the parameter was significantly lower in group A: 15,713.5 \pm 9003.5 vs. group B: $26,238.2 \pm 8506.5 \mathrm{mg}^{\star} \mathrm{min} / \mathrm{dl}(\mathrm{p}=0.02)$ (Figure 2).

\section{Postprandial glucose excursion}

We noticed statistically significant differences in PPGE. This was $47.4 \pm 39.8$ and $76.2 \pm 58.2 \mathrm{mg} / \mathrm{dL}$ for groups A and B respectively $(\mathrm{p}=$ 0.03 ). The time needed to reach the glycaemic peak was similar in both groups (approx. 55 minutes, $\mathrm{p}=0.83$ ).

\section{Postprandial hyperglycaemia}

The frequency of postprandial hyperglycaemia during 180 minutes did not reach the level of statistical significance $(\mathrm{p}=0.45)$. RR was $73 \%$ and NNT 7 (Table 3).

\section{Postprandial hypoglycaemia}

The hypoglycaemia episodes in capillary blood over a 120-minute period occurred occasionally in both groups. Moreover, there was no statistically significant difference between the groups in CGMS over the 180-minute observation period $(\mathrm{p}=0.98)$ (Table 4$)$. 
Citation: Groele L, Golicki D, Błazik M, Pańkowska E (2014) Improving the Estimation of Meal-Time Insulin Dose Based On the Glycaemic Load of a Meal in Children with Type 1 Diabetes on Insulin Pump Therapy: A Randomized Study. J Diabetes Metab 5: 435 doi:10.4172/21556156.1000435

Page 4 of 5

\section{Comparison of glucose measured methods}

In group A from 30 to 120 minutes, and in Group B from 30 to 90 minutes after the meal, the value of glycaemia measured in capillary blood was significantly higher than that measured by CGMS( $\mathrm{p}<0.05)$ (Figures 3 and 4 ).

\section{Discussion}

The analysis presented shows that increased ICR for a meal with a

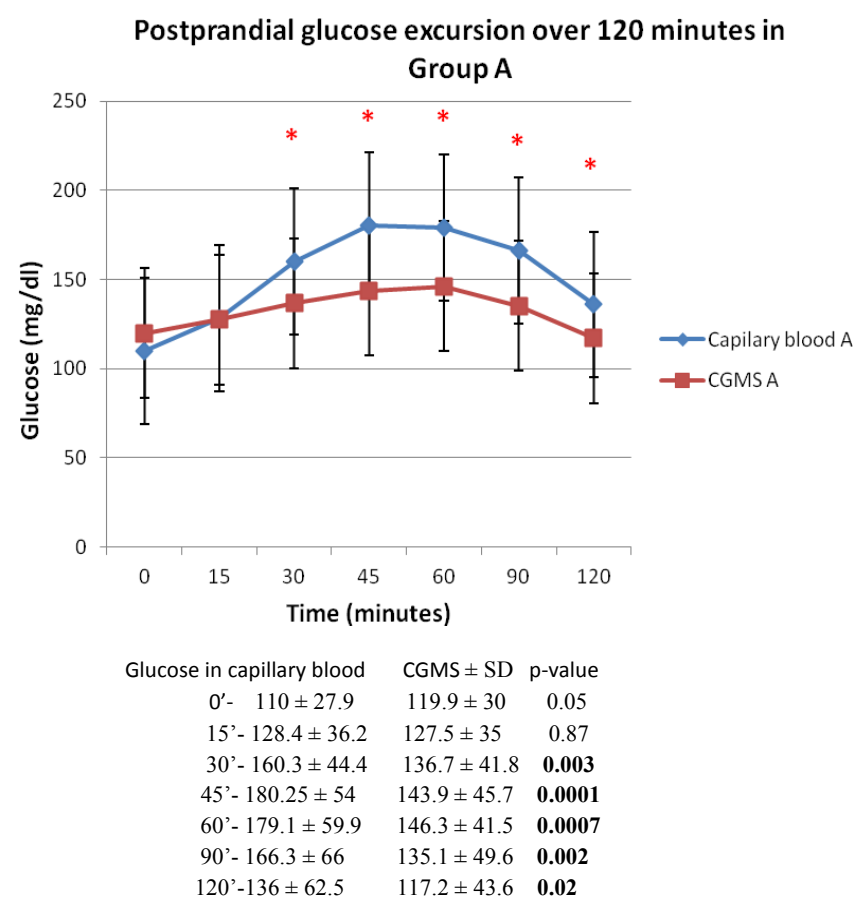

Figure 3: Postprandial glucose excursion over 120 minutes in accordance with the measurement method in Group A.

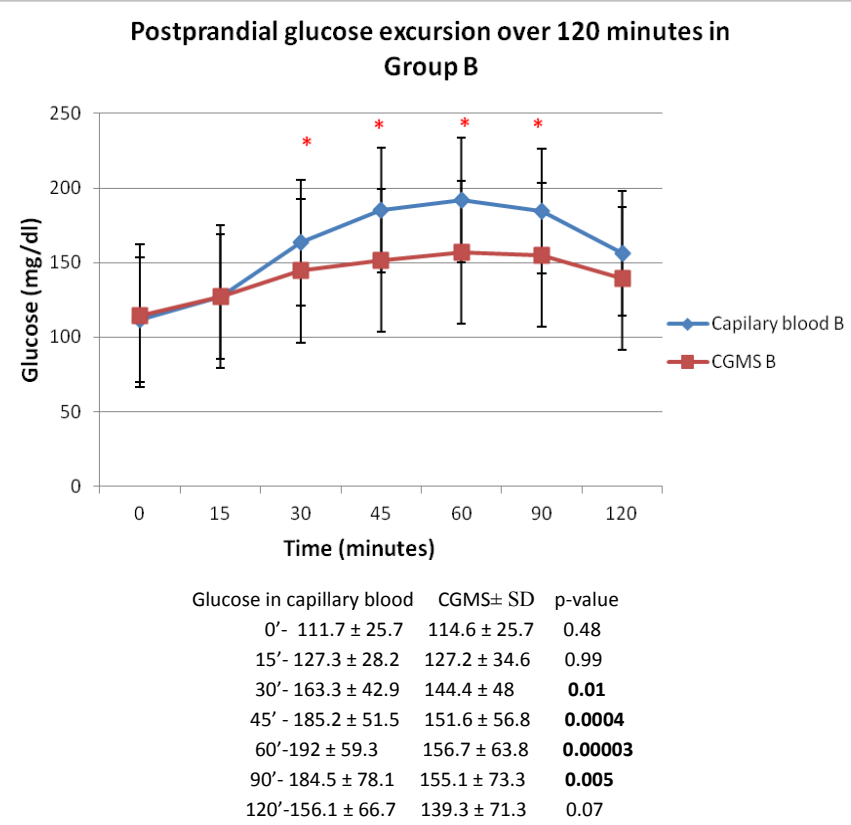

Figure 4: Postprandial glucose excursion over 120 minutes in accordance with the measurement method in Group B. high GL decreased the PPGE. We noted significantly lower postprandial glycaemic changes in the experimental group $\mathrm{A}$, where the insulin dose was increased by $30 \%$ compared to a standard dose used in group B. In group A, the hyperglycaemic peak was significantly lower than in the control group $(\mathrm{p}=0.03)$. Moreover, the increased amount of insulin did not lead to greater hypoglycaemic risk. When assessing the PPGE, the peak of hyperglycaemia was significantly higher when it was estimated in capillary blood than when measured by the CGM in the interstitial fluid.

It is interesting to compare our results with the results of studies conducted in healthy people by Brand-Miller et al. [11] and by Galgani in obese women [12]. These studies show how a high glucose load meal causes an increased response in insulin and glucose levels. The authors presented the PPGE as AUC data. Brand-Miller showed the maximum glucose level at a peak of $52 \mathrm{mg} / \mathrm{dL}$ after $50 \mathrm{~g}$ of carbohydrates from breakfast cereals in healthy people [9]. In our study, when intervention was implemented, the peak glucose level was $47.5 \mathrm{mg} /$ dL. We can conclude that increased insulin doses seem to cause higher concentrations of insulin during the hyperglycaemic peak than the standard insulin ratio averaged for all kind of carbohydrate products. O'Connell, who conducted a study in young people with type 1diabetes, showed a significant upward tendency in postprandial glycaemia with a greater glucose response (AUC) when the insulin was applied in a standard or dual-wave bolus for a high glycaemic index meal than for a low glycaemic index meal [13]. In O'Connell's study, the maximum glucose level in type 1 diabetes patients (CGMS) was $95 \mathrm{mg} / \mathrm{dL}$, while in healthy participants it was $32.4 \mathrm{mg} / \mathrm{dL}$ [13]. The time needed to reach the maximum glucose level in our study was 55 minutes, which is comparable to the results of a study in type 2 diabetes patients [14]. However, it was shorter than in the study by O'Connell et al, where it was 76 minutes for type 1 diabetes patients [13]. In healthy people, the mean time spent to reach peak glucose level is shorter and closer to 30 minutes $[9,15]$. Other studies regarding type 1 diabetes children highlight the impact of the glucose index of a meal on the PPGE. One of them, by Mohammed et al., proved that the changes in glucose AUC are related to GI [6].

Our findings showed a relative risk of hyperglycaemia reduction at $22 \%$ in the experimental group. In an analysis by Lippaiova et al. [16], the mean weekly glycaemia measured by CGMS before, during and after a high load meal did not differ, but the mean glucose level did not reflect changes which appear after the meal.

Our study showed that products with a high GL require a higher dose of insulin to decrease the PPGE and the postprandial glycaemic response in children between 8-12 years. The efficiency of our intervention can influence daily glucose fluctuation related to meals in patients with type 1 diabetes mellitus. Moreover, it has an impact on decreasing oxidative stress and protein glycation.

When considering the results of capillary blood measurements and CGMS outcomes, we did not observe any statistically significant differences in the frequency of hypoglycaemic episodes. During the observation period up to 180 minutes after the meal and bolus administration, we did not observe any signs of hypoglycaemia. There were no severe hypoglycaemic episodes. These results are comparable with the findings of O'Connell, who presented a similar frequency of hypoglycaemic episodes independently of the type of bolus [13]. In Giacco's study, the authors observed that consuming a high GL meal corresponds to a higher risk of hypoglycaemia than a low GL meal [17].

Using two methods for estimating glucose levels, we observed relevant differences in their values critical at 30, 90, 120 minutes after the meal. This phenomenon has been described by other researchers 
Citation: Groele L, Golicki D, Błazik M, Pańkowska E (2014) Improving the Estimation of Meal-Time Insulin Dose Based On the Glycaemic Load of a Meal in Children with Type 1 Diabetes on Insulin Pump Therapy: A Randomized Study. J Diabetes Metab 5: 435 doi:10.4172/21556156.1000435

Page 5 of 5

[18]. Nowadays, CGMS is the dominant method of glucose value estimation. In the light of our results and other research results, we would like to open a discussion on the reliability of post-meal glucose level data obtained only from CGMS. CGMS is a valuable method to assess glucose trends, but it does not accurately reflect glucose levels in the blood. On the other hand, it allows the identification of overlooked hyper- or hypoglycaemia [19]. After various types of food consumption, glycaemia remains above the baseline for a longer period, and the CGMS is a convenient method to determine the glucose response. We observed this in the post-meal reaction over 180 minutes, which was a compatible with the result obtained by Chlup [20].

The strength of this trial involves the size of the group, the methodology employed and the use of the 'gold standard' method in measuring glucose levels. Randomisation with stratification according to age decreased the risk of bias in enrolling the study group. Precise inclusion and exclusion criteria lead to the selection of homogenous groups of participants.

Unfortunately, the strength of this trial was limited by its length. This was a short-term intervention conducted on one day only. The meal with a high glycaemic load was not tested on subsequent days. We did not experiment with other products with high GL, either. Our study showed, for the first time, the beneficial effects of increased insulin doses administered before high GL meals on PPG. Thus not only the carbohydrate-insulin ratio but also the glycaemic load-insulin ratio should be used to calculate the insulin dose before meals. This analysis highlights a number of important practical solutions for those who take care of patients with type 1 diabetes on insulin pump therapy. Our findings could influence diabetes management. This does not mean that we wish to promote a high GL diet. The harmful metabolic effects of such food on healthy or diabetic people are commonly known. Although increased doses of meal insulin allow better glucose control to be achieved, consumption of meals with high GL should be limited. Additional augmentation of insulin doses for high GL meals could imply greater insulin resistance with all its consequences. On the other hand, the efficiency of the above-mentioned intervention could essentially decrease the risk of postprandial hyperglycaemia. These findings should be supported by longitudinal observations. It is known that in order to achieve the maximum effect on postprandial glycaemia levels and to reduce hypoglycaemia, rapid analogues should be injected even 20 minutes prior to meals [7,21]. This is not commonly done by the majority of pump users [22]. In a pilot study [8], devices which accelerate insulin absorption by heating up the injection site decreased postprandial glucose concentrations. The question remains whether the patients will use this technique in everyday life.

Furthermore, the issue of different meal portions among groups must be explored. All the groups should receive a high glycaemic load meal for breakfast, but not the same portion size, because of the different caloric demands depending on age. It is worth emphasising that we assessed the body's reaction only to the cereal and not to milk; that is why in the experimental group A, the ICR increased by $30 \%$ was applied only to breakfast cereal. The insulin dose for milk was calculated using standard individualised ICR in both groups.

To conclude, this study raises an issue which is very important but difficult to resolve, which healthcare providers encounter on an everyday basis. Finding the optimal answer is virtually impossible because of the well-known harmfulness of a high glycaemic load diet, but our findings may help to decrease its adverse effects.

\section{References}

1. American Diabetes Association (2013) Standards of medical care in diabetes--2013. Diabetes Care 36 Suppl 1: S11-66.
2. Bangstad HJ, Danne T, Deeb L, Jarosz-Chobot P, Urakami T, et al. (2009) Insulin treatment in children and adolescents with diabetes. Pediatr Diabetes 12:82-99.

3. Group DS (2002) Training in flexible, intensive insulin management to enable dietary freedom in people with type 1 diabetes: dose adjustment for normal eating (DAFNE) randomised controlled trial. British Journal of Nutrition.

4. Carbohydrates in human nutrition. Report of a Joint FAO/WHO Expert Consultation. FAO Food Nutr Pap 66: 1-140.

5. Australia S. The glycemic index of foods. Available from http://www.sai global. com/shop/scripts/Details.asp?DocN=ASO733779662AT 2007.

6. Mohammed NH, Wolever TM (2004) Effect of carbohydrate source on postprandial blood glucose in subjects with type 1 diabetes treated with insulin lispro. Diabetes Res Clin Pract 65: 29-35.

7. Cobry E, McFann K, Messer L, Gage V, VanderWel B, et al. (2010) Timing of meal insulin boluses to achieve optimal postprandial glycemic control in patients with type 1 diabetes. Diabetes Technol Ther 12: 173-177.

8. Raz I, Weiss R, Yegorchikov Y, Bitton G, Nagar R, et al. (2009) Effect of loca heating device on insulin and glucose pharmacokinetic profiles in an openlabel, randomized, two-period, one-way crossover study in patients with type 1 diabetes using continuous subcutaneous insulin infusion. Clinical Therapeutics 31:980-987.

9. Brand-Miller JC, Stockmann K, Atkinson F, Petocz P, Denyer G (2009) Glycemic index, postprandial glycemia, and the shape of the curve in healthy subjects: analysis of a database of more than 1,000 foods. Am J Clin Nutr 89: 97-105.

10. Elizabeth R. Seaquist, John Anderson, Belinda Childs, Philip Cryer, et al (2013) Hypoglycemia and Diabetes: A Report of a Workgroup of the American Diabetes Association and The Endocrine Society Diabetes Care 36:1384-1395.

11. Brand-Miller JC, Thomas M, Swan V, Ahmad Zl, Petocz P, et al. (2003) Physiological validation of the concept of glycemic load in lean young adults. $J$ Nutr 133: 2728-2732

12. Galgani J, Aguirre C, Díaz E (2006) Acute effect of meal glycemic index and glycemic load on blood glucose and insulin responses in humans. Nutr J 5: 22

13. O'Connell MA, Gilbertson HR, Donath SM, Cameron FJ (2008) Optimizing postprandial glycemia in pediatric patients with type 1 diabetes using insulin pump therapy: impact of glycemic index and prandial bolus type. Diabetes Care 31: 1491-1495

14. Ionescu-Tarquoviste C, Popa E, SÃßntu E, Mihalache N, CheÅ£a D, et al (1983) Blood glucose and plasma insulin responses to various carbohydrates in type 2 (non-insulin-dependent) diabetes. Diabetologia 24: 80-84.

15. Nilsson A GY, Ostman E, Preston T, Bjorck I (2006) Effects of GI and content of indigestible carbohydrates of cereal-based evening meals on glucose tolerance at a subsequent standardised breakfast. European Journal of Clinical Nutrition 60:1092-1099.

16. Lippaiova N PM, Kuzmina G, Peterson K, Fajkosova L, Luza J (2008) Safety of New algorithms for premeal insulin boluses in high glycaemic index meals in persons with type 1 diabetes mellitus Rusing insulin pumps. Biomed Pap Med Fac Palacky Olomouc Czech Republic 152:73-77.

17. Giacco R PM, Rivellese AA, Lasorella G, Giacco A, D’Episcopo L, (2000) Long-term dietary treatment with increased amounts of fiber-rich low glycemic index natural foods improves blood glucose control and reduces the number of hypoglycemic events in type 1 diabetic patients. Diabetes Care 23:1461-1466.

18. Djakouré-Platonoff C, Radermercker R, Reach G, Slama G, Selam JI (2003) Accuracy of the continuous glucose monitoring system in inpatient and outpatient conditions. Diabetes Metab 29: 159-162.

19. Melki V, Ayon F, Fernandez M, Hanaire-Broutin H (2006) Value and limitations of the Continuous Glucose Monitoring System in the management of type 1 diabetes. Diabetes Metab 32: 123-129.

20. Chlup R PK, Zapletalova J, Kudlova P, Seckar P (2010) Extended prandia glycemic profiles of foods as assessed using continuous glucose monitoring enhance the Power of the 120-minute glycemic index. Journal of Diabetes Science and Technology 4:615-624.

21. Gough SC (2007) A review of human and analogue insulin trials. Diabetes Res Clin Pract 77: 1-15.

22. Heinemann L (1995) Do insulin-treated diabetic patients use an injection-mealinterval in daily life? Diabet Med 12: 449-450. 